\title{
Personalized cancer vaccines show glimmers of success
}

Treatments tailored to a person's individual cancer mutations train immune system to attack tumours.

\section{Heidi Ledford}

05 July 2017

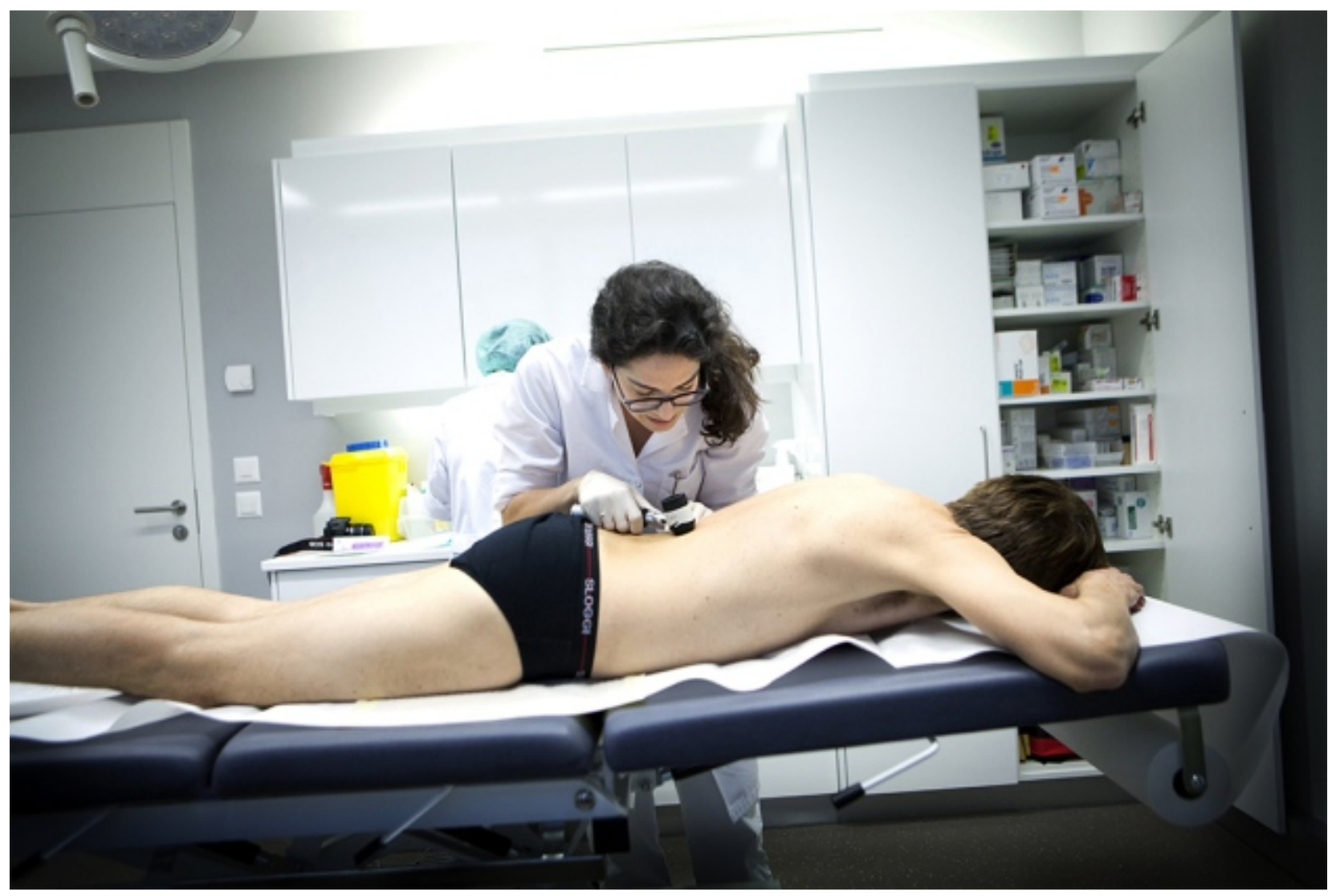

BSIP/UIG via Getty Images

Melanoma cancers seem receptive to genetically tailored treatments that harness the immune system.

Vaccines tailored to match a person's particular constellation of cancer mutations seem to have fended off tumours in a handful of patients, two small clinical trials show.

The vaccines are described in papers published in Nature on 5 July ${ }^{1,2}$. The studies are the first to report that the approach — which is gaining support in academia and industry - could combat cancer 
in humans. They also provide hints about ways to boost the vaccines' power by combining them with treatments that target the immune system, called immunotherapies.

"It's potentially a game changer," says Cornelis Melief, a cancer immunologist at Leiden University Medical Centre in the Netherlands, and author of a commentary accompanying the papers. "The two papers really strongly indicate that the patients experienced clinical benefit."

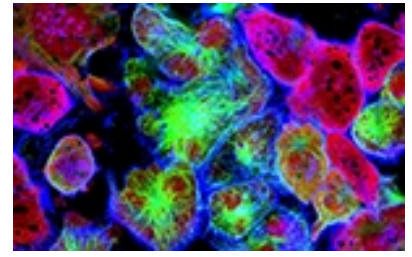

DNA typos to blame for

In principle, the vaccines are similar to those used against infectious most cancer mutations diseases: unique components of a foreign invader - cancer cells, in this case - are mixed with agents that stimulate an immune response. The mixture is injected into the patient, in the hope of triggering an immune attack strong enough to vanquish the invader.

But for personalized cancer vaccines, the components are tailored to each patient and are administered after the cancer has already appeared — rather than aiming to prevent occurrence.

\section{Looking for a response}

In the studies, researchers began by sequencing the genes that encode proteins in each patient's tumour. They selected mutant proteins that were most likely to generate an immune response and used these as the basis for their vaccines.

One group, led by Catherine Wu at the Dana-Farber Cancer Institute in Boston, Massachusetts, treated six people with melanoma, a type of skin cancer. For each person, they

\section{Related stories}

- Algorithms compete to predict recipe for cancer vaccine

- Researchers push for personalized tumour vaccines

- Cocktails for cancer with a measure of immunotherapy

More related stories

\section{Related stories}

- Algorithms compete to predict recipe for cancer vaccine

- Researchers push for personalized tumour vaccines

- Cocktails for cancer with a measure of immunotherapy

More related stories formulated a vaccine that contained up to 20 protein fragments corresponding to the mutations in their tumours ${ }^{1}$. The participants, who received surgery to remove their tumours, had been deemed at high risk for cancer recurrence. But they were not due to receive further treatment unless their cancer came back. Recurrence typically occurs in about half of all such melanoma patients, says Wu. 
Two years later, four of those patients had not seen their tumours return. And although tumours did grow in the remaining two participants, both experienced a complete remission when subsequently treated with a drug that rouses the immune system by blocking a protein called PD-1.

The second group, led by Ugur Sahin, a physician who studies tumour immunology and cancer genomics at the University of Mainz in Germany, treated 13 melanoma patients with vaccines that contained RNA encoding up to 10 mutated proteins in each patient ${ }^{2}$. The eight patients who had no visible tumours at the time of vaccination remained tumour-free more than a year later.

The remaining five participants' tumours had spread by the time they received the vaccine. Tumours shrank in two of them, but later resurged in one patient. A third experienced a complete remission after subsequent treatment with a PD-1 inhibitor.

\section{Challenges ahead}

Personalized cancer vaccines had already been shown to provoke immune

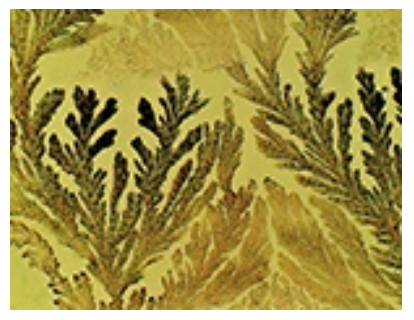

Cancer therapy: an evolved approach responses in humans ${ }^{3}$. But the new trials are the first to evaluate whether these immune responses can successfully battle tumours. The numbers are small and the trials lacked a control group, but the results are encouraging, says Robert Schreiber, a cancer immunologist at Washington University in St Louis, Missouri. Larger trials in academia and industry are ongoing, he notes, and researchers are particularly interested in combining the vaccines with PD-1 inhibitors. "I'm convinced that personalized vaccines are a way to go," he says.

The field still faces challenges. It took both teams about three months to formulate and manufacture their vaccines - too long to delay treatment for many cancers. But both groups say they could accelerate the process as they scale it up. Wu estimates that she could get the lag down to six weeks.

It is also unclear how many types of cancer will respond to the approach. Cancer vaccines may work best if they target several different cancer mutations, to lessen the chance of a tumour becoming resistant to the vaccine by shedding any one particular mutation. The genomes of melanoma cells tend to carry many mutations, giving researchers a host of options when designing the vaccines. But some cancers will present fewer avenues for attack. "We have to think about how we can launch a multipronged attack," says Wu. "How many prongs do you need? We don’t know." 
With a wave of clinical trials in the pipeline, researchers will gradually work out which cancers are suited to the approach, and how best to combine the vaccines with other treatments, says Sahin. "We are entering the next phase of rational cancer immunotherapy," he says.

Nature doi:10.1038/nature.2017.22249

Read the accompanying News \& Views article: 'Precision T-cell therapy targets tumours'

\section{References}

1. Ott, P. A. et al. Nature http://dx.doi.org/10.1038/nature22991 (2017).

2. Sahin, U. et al. Nature http://dx.doi.org/10.1038/nature23003 (2017).

3. Carreno, B. M. et al. Science 348, 803-808 (2015). 